\title{
The Value of Patient-Provider Interactions in Orthopedic Settings: An Evidence-to-Practice Review
}

Matthew S. Panetta, SCAT, ATC; Carly J. Wilson, SCAT, ATC; and Zachary Winkelmann, PhD, SCAT, ATC

University of South Carolina, Columbia, SC

\begin{abstract}
Clinical outcomes are influenced by specific physical therapy interventions and several nonspecific factors associated with the healthcare professional, patient, and setting. The relationship built between patients and providers is based on treatment outcomes is referred to as the patient-provider interaction, which is a nonspecific factor. The purpose of this review was to investigate patients' and clinicians' perceptions of factors that influence patient-provider interactions. Eleven different databases were accessed as potential research sources. Thirteen qualitative studies were selected that examined the perceptions of non-specific factors which impact the patient-clinician relationship as well as the perceptions that healthcare providers and patients in musculoskeletal settings. Out of these 13 articles, four common themes were found to influence the patient-provider interaction across all the literature selected for this review: clinician interpersonal and communication skills, clinician practical skills, individualized patient-centered care, organizational and environmental factors. Articles for this study were only considered for review if they were published in English. This was stated as a limitation of the study, as well as the fact that this review only identified factors that are perceived to be related to patient-provider interactions. The review highlighted that patients and clinicians believe communication, interpersonal and practical skills, individualized care, and appropriate time and flexibility for patient care influence patient-provider interaction in musculoskeletal settings. These factors can serve as facilitators as well as barriers, depending on the level of intensity that they are implemented in the overall patientprovider interaction. Athletic trainers, as healthcare professionals, need to be responsible for implementing patient-centered care concepts to encourage a healthy patient-provider interaction.
\end{abstract}

Key Phrases

Organizational and personal outcomes \& patient education

Correspondence

Dr. Zachary Winkelmann, University of South Carolina

1300 Wheat Street, Columbia, SC 29208.

E-mail:winkelz@mailbox.sc.edu

Twitter: @zachwinkelmann

Copyright (C) by Indiana State University All rights reserved. ISSN Online 2577-8188

\section{Full Citation}

Panetta MS, Wilson CJ, Winkelmann ZK. The value of patient-provider interactions in orthopedic settings: an evidence-to-practice review. Clin Pract Athl Train. 2020;3(3): 18-23.

https://doi.org/10.31622/2020/0003.3.4.

Submitted: April 20, 2020 Accepted: September 1, 2020

\section{ORIGINAL REFERENCE AND SUMMARY}

O'Keeffe M, Cullinane P, Hurley J, Leahy I, Bunzli $S$, O'Sullivan $P$, O'Sullivan $K$. What influences patient-therapist interactions in musculoskeletal physical therapy? Qualitative systematic review and meta-synthesis. Phys Ther. 2016;96(5):609-622.

\section{SUMMARY}

\section{CLINICAL PROBLEM AND QUESTION}

$S_{\text {ports medicine providers assist a wide range }}$ of patients with various degrees of musculoskeletal pain, guiding them through both the physical and psychological aspects of recovery and rehabilitation and often forming strong bonds along the way. Healthcare providers who practice from a clinician-driven mindset will focus on identifying the diagnosis and prescribing interventions and rehabilitation for treatment. ${ }^{1} \mathrm{~A}$ clinician-driven mindset can often cause the provider to neglect important patient-centered care principles during the patient-provider interaction. The patient-provider interaction is defined as the sense of collaboration, warmth, and support between the patient and the clinician. ${ }^{2}$ Previous research has focused on the impacts that patient-provider relationships have on treatment outcomes. The common theme identified in multiple studies has been a strong

Clinical Practice in Athletic Training Volume 3 - Issue 3 - November 2020 
bond between the healthcare provider and patient results in improved patient outcomes and recovery. ${ }^{3}$ Positive patient interactions in therapeutic rehabilitation settings have been linked to reduced pain and disability, as well as higher treatment satisfaction. ${ }^{4}$ However, there is little known about what specific components ultimately help form and facilitate that crucial relationship between the patient and healthcare provider. The primary clinical question of this systematic review was to explore what factors hindered or assisted the relationship formed between sports medicine providers and their patients through the therapeutic rehabilitation process. The research team of this systematic review focused on the term "nonspecific factors", defined as factors associated with provider, patient, and setting, to differentiate from more clinically related factors such as prescribed interventions.

\section{SUMMARY OF LITERATURE}

Previous literature selected for the guiding systematic review ${ }^{2}$ had to meet specific guidelines based on the clinical question. Studies were included if they examined the opinions and viewpoints of patients or physical therapists in regard to the factors that proved to enable or serve as an obstacle to a positive interaction between patients and their provider. ${ }^{2}$ Exclusion criteria was defined as primarily quantitative studies, literature that was not reported in English, studies looking at settings that did not fit musculoskeletal physical therapy or conditions, or measured only the strength of the relationship between healthcare provider and patient rather than the factors that established it in the first place. ${ }^{2}$ Through quality assessment and screening, 13 articles were approved to be included in the systematic review and meta-synthesis with a total of 253 patients and 78 providers being interviewed. The guiding systematic review ${ }^{2}$ was the first to look at provider and patient perceptions of patient-centered care simultaneously. Eight articles investigated clinician's interpersonal and communication skills, ten articles evaluated clinician practical skills, seven articles examined individualized patientcentered care, and six articles investigated organizational and environmental factors. ${ }^{2}$

\section{SUMMARY OF OUTCOMES}

The authors from the guiding systematic review ${ }^{2}$ examined the providers' and patients' perceptions of factors that influence patient-provider interactions in musculoskeletal settings. The authors examined the thirteen qualitative studies using the Critical Appraisal Skills Programme (CASP) Qualitative Research Assessment Tool. The patient-provider interaction is also referred to as the relationship between patients and healthcare providers on treatment outcomes. The relationship is usually built from a sense of collaboration, warmth, and support between individuals and includes agreement on goals and interventions and overall attitudes among each other. ${ }^{2}$ This is essentially the basis of patient-centered care: ensuring that high quality, holistic care is being provided that includes the values and goals of the patient.

\section{FINDINGS AND CLINICAL IMPLICATIONS}

From the 13 studies that were included, four themes were reported to influence patientclinician interactions (Figure 1). The first theme encompassed clinician interpersonal skills, such as active listening, empathy, friendliness, encouragement, confidence, and nonverbal communication. ${ }^{2}$ Physical therapist practical skills comprised the second theme, which focused on clinician practical skills, which included proficiency, training and ability to explain healthcare concepts to the patient. Theme three was individualized patient-centered care, which was assessed by taking the patient's opinion into consideration and individualizing the treatment. Lastly, organizational, and environmental factors, 


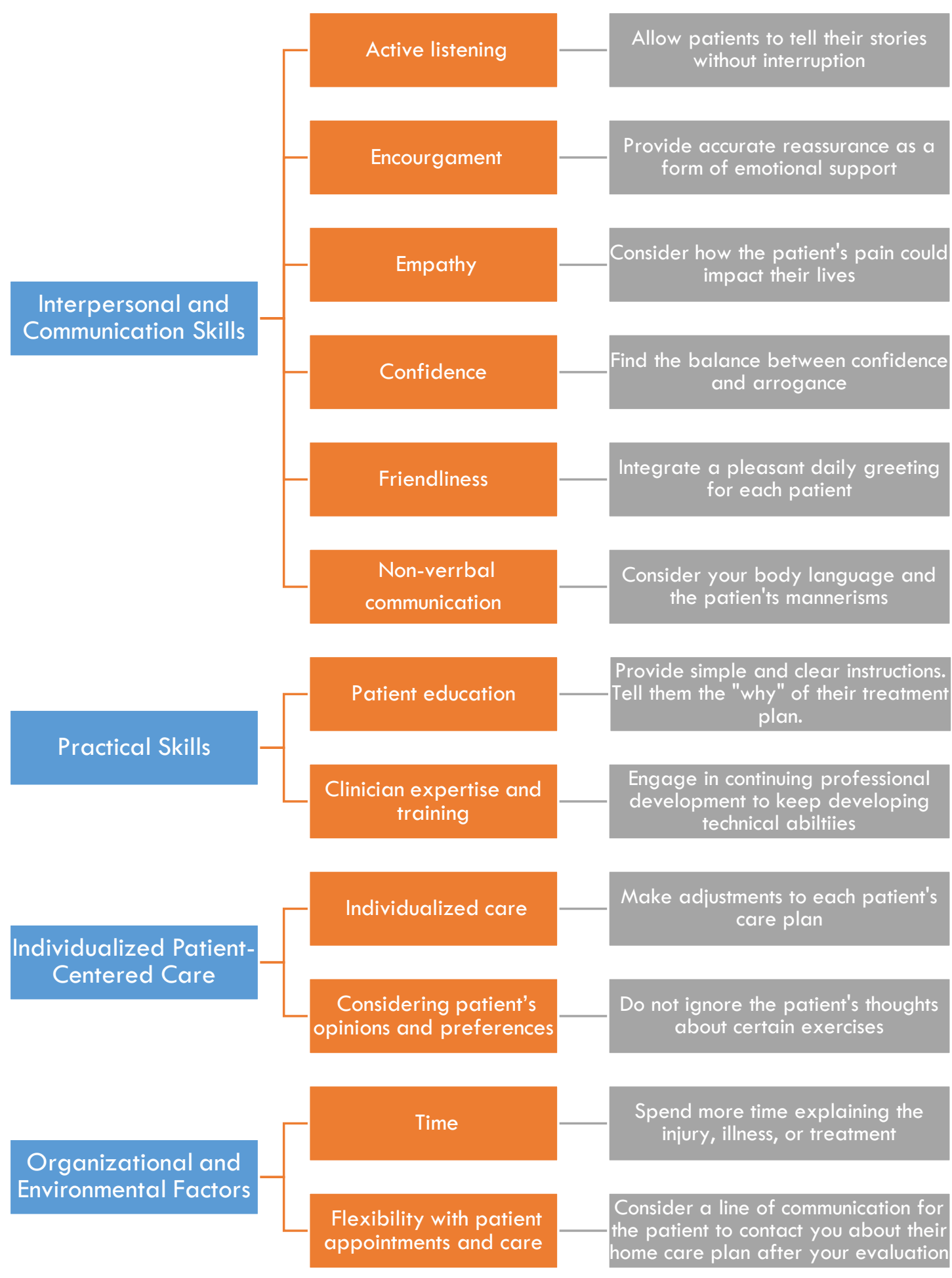

Figure 1. Main Influences of Patient-Provider Interactions in Musculoskeletal Physical Therapy. The first and second column are the main themes and traits from the study. The third column includes clinical bottom lines and suggestions to improving patient-provider interactions.

Copyright $\left({ }^{\circ}\right.$ by Indiana State University All rights reserved. ISSN Online 2577-8188 Volume 3 - Issue 3 - November 2020 
including the time and provider's flexibility with care and appointments constituted the fourth theme. The four themes found in this study can be incorporated regularly into everyday athletic training clinical practice to enhance patientprovider relationships.

Theme 1 - Clinician Interpersonal and Communication Skills

There were several interpersonal and communication factors from both the patient and clinician that determined the quality of interactions, such as verbal and nonverbal communicative ability, empathy, and trust. Active listening was the most common aspect stated by both patient and clinician. ${ }^{2}$ Active listening is giving one's undivided attention to the speaker while using appropriate non-verbal communication to assure the patient that they are being understood. ${ }^{2}$ Clinicians who actively listen will reassure the patient that they are valued, which will strengthen the patient-clinician bond. 5 The findings also showed a discrepancy between clinician and patient about encouragement for the patient during their appointment. Patients that did not receive enough encouragement may feel less motivated to give their best effort during rehabilitation and improvement goals may not be met on time. ${ }^{6}$ From the thirteen articles included in the guiding review, there were zero reported statements regarding encouragement from the clinician's stance. Athletic trainers should practice active listening with non-verbal communication, along with sincere empathy and encouragement for the patient to optimize patient satisfaction.

\section{Theme 2 - Clinician Practical Skills}

The next theme focused on the practical skills of the clinician. Healthcare professionals and patients share the belief that healthcare expertise was crucial to the development of a strong patient-clinician relationship. ${ }^{2,6}$ Clinician expertise encourages patients to develop trust and reliance with the clinician, strengthening the patient-

Copyright $(\subseteq$ by Indiana State University All rights reserved. ISSN Online 2577-8188 provider interaction. ${ }^{2}$ Clinicians stated that continuing education and skill development were important to the maintenance of the patientclinician relationship. Patients highly valued a provider who could easily explain the patient's problem, how the provider could help them, and why the provider was prescribing specific exercises in their rehabilitation. 2,7 The concept of patient education can relate directly back to athletic training, where the athletic trainer is on the front lines of sharing and disseminating information to the patient on their diagnosis and rehabilitation plan while avoiding medical jargon. Athletic trainers should address health literacy and assist patients in their capacity to process and understand their health conditions. Patient education with clear explanations can help the patient feel more comfortable with their rehabilitation protocol and clinician's decision making. ${ }^{2}$ Athletic trainers share this priority for developing and maintaining clinical competency and have expressed a professional desire for more research to be done in focus group sessions for the Prioritized Research Agenda for the Athletic Training Profession organized by the Strategic Alliance Research Agenda Task Force. ${ }^{8}$

Theme 3 - Individualized Patient-Centered Care

Patient-centered care is the practice of being "respectful of and responsive to individual patient preferences, needs, and values, and ensuring that patient values guide all clinical decisions." 9 Patients highlighted the importance of individualized patient care and taking into consideration patients' opinions and preferences in regards to the patient-provider relationship ${ }^{2}$, stating that they found it annoying when their clinician ignored their preferences and felt that it diminished the overall interaction. ${ }^{5}$ Patients appreciated when their clinician made an effort to adjust or adapt their rehabilitation in relation to their inability to complete a task, which positively affected the patient-provider relationship. ${ }^{2}$ This can easily relate to the 
profession of athletic training. Athletic trainers are providers with the unique opportunity to work with patients from initial injury to final discharge. Throughout this process, the athletic trainer should tailor the patient's rehabilitation to their functional goals and personal preferences to facilitate patient engagement and commitment.

Theme 4 - Organizational and Environmental Factors

A healthcare professional's lack of organization regarding the amount of time given to patients and appointment flexibility created a general dissatisfaction for patients. ${ }^{2}$ Athletic trainers may be limited in their amount of time for patient care. In this guiding review, both patients and providers stated that allowing patients appropriate time to explain their problem and discuss their treatment was essential to maintaining a positive patientprovider interaction. ${ }^{2}$ It may be beneficial for the provider to be proactive and plan out each patient's rehabilitation and main points of discussion prior to seeing them, as well as being flexible. However, only one healthcare provider in the guiding review stated that flexibility with patient appointments and care was important, compared to five patient statements. Patients expressed appreciation towards clinicians who accommodated patient care and appointment scheduling based on their needs. ${ }^{2}$ Athletic trainers work with a variety of patient populations who also have busy schedules that have to be worked around to fit in rehabilitation appointments. To reduce the feeling of being rushed and increase the patient's satisfaction, athletic trainers should outline the allotted time available for the patient's session at the beginning of the appointment. ${ }^{10}$ By implementing patient-centered care techniques into appointment scheduling, athletic trainers can maximize the use of their time and convey that the patient's time is just as valued.

\section{CLINICAL BOTTOM LINE}

Copyright (C) by Indiana State University All rights reserved. ISSN Online 2577-8188
The guiding review concluded that healthcare professionals and patients believe that communication and interpersonal skills, practical skills, individualized care, and organizational and environmental factors have influences on the relationship developed during the rehabilitation for musculoskeletal injuries. Furthermore, there is a difference between clinicians and patients about the value of patient education. Patients rated their own education of their rehabilitation as highly important to the facilitation of the patientprovider interaction, however clinicians rated it low in value. Patients primarily felt importance in active listening, patient education, individualized rehabilitation, and encouragement from their clinician. Clinicians that take the time to actively listen and provide individualized care are much more likely to develop stronger interactions and relationships that may promote rehabilitation outcomes.2,5-7 Scheduling out a block of time during the day for one patient to come in while preventing interruptions from other individuals can show the patient that the clinician has their undivided attention for communication and rehabilitation. $5,6,11$

Patients value their feelings and beliefs regarding their care planning. Similarly, athletic trainers' value their own time and effort that they put into treatment and rehabilitation with their patients. Patients in the guiding review reported strong values in education from the clinician. Athletic trainers with a sufficient understanding of the injury and patient's goals can enhance the patientclinician interaction through patient-centered care tactics. Gaining patient insight can be accomplished by asking what questions or worries the patient may be having about their rehabilitation process. Athletic trainers should also work on creating a habit in providing clear explanations of their responsibilities and duties with the patient when creating goals and decision making with certain interventions. The guiding review also observed that patients reported that encouragement from their clinician was important,

Clinical Practice in Athletic Training Volume 3 - Issue 3 - November 2020 
even though the clinician did not see any value in this factor. Encouraging patients through their rehabilitation exercises demonstrates a deeper level of care and emotional support, which aligns specifically with patient-centered care principles. The evidence from this review supports clinician interpersonal and practical skills, individualized care, and organization factors as influences that can cultivate the patient-clinician relationship.

\section{REFERENCES}

1. Bensing J. Bridging the gap: The separate worlds of evidence-based medicine and patient-centered medicine. Patient Educ Couns. 2000;39(1):17-25. https://doi.org/10.1016/S07383991 (99)00087-7.

2. O'Keeffe M, Cullinane P, Hurley J, et al. What influences patient-therapist interactions in musculoskeletal physical therapy? Qualitative systematic review and meta-synthesis. Phys Ther. 2016;96(5):609-622. https://doi.org/10.2522/pti.20150240

3. Rathert C, Wyrwich M, Boren S. Patientcentered care and outcomes: a systematic review of the literature. Med Care Res Rev. $2013 ; 70(4): 351-379$. https://doi.org/10.1177/1077558712465 774.

4. Ferreira P, Ferreira $M$, Maher $C$, Refshauge K, Latimer J, Adams R. The therapeutic alliance between clinicians and patients predicts outcome in chronic low back pain. Phys Ther. 2013;93(4):470-478. https://doi.org/10.2522/pti.20120137.

5. Potter $M$, Gordon S, Hamer P. The physiotherapy experience in private practice: the patients' perspective. Aust J Physiother. 2003;49(3): 195-202.

https://doi.org/10.1016/S00049514(14)60239-7.

6. Gyllensten A, Gard G, Hansson L, Ekdahl C. Interaction between patient and physiotherapist in psychiatric care - the

Copyright $\left({ }^{\circ}\right.$ by Indiana State University All rights reserved. ISSN Online 2577-8188 physiotherapist's perspective. Adv Physiother. 2000;2(4):157-167.

https://doi.org/10.1002/pri.156

7. Kidd M, Bond C, Bell M. Patients' perspectives of patient-centeredness as important musculoskeletal physiotherapy interactions: a qualitative study. Physiotherapy. 2011 ;97:154-162.

https://doi.org/10.1016/i.physio.2010.08.0 02.

8. Eberman LE, Walker SE, Floyd R, Covassin T, Nolton E, Valier A, Phan K, Weiss L, EarlBoehm J. The prioritized research agenda for the athletic training profession: A report from the Strategic Alliance Research Agenda Task Force. J Athl Train. 2019;54(3):237-44. https://doi.org/10.4085/1062-6050-37418.

9. Richardson W, Berwick D, Bisgard J, Bristow L, Buck C, Cassel C. Crossing the quality chasm: a new health system for the $21^{\text {st }}$ century: Institute of Medicine. Washington: National Academy Press; 2001. https://doi.org/10.17226/10027.

10. Smith R. Patient-centered interviewing. 2nd ed. Philadelphia: Lippincott Williams \& Wilkins, 2002.

11. Hills R, Kitchen S. Satisfaction with outpatient physiotherapy: focus groups to explore the views of patients with acute and chronic musculoskeletal conditions. Physiother Theor Pr.2007;23(1): 1 -20.

https://doi.org/10.1080/0959398060102 3705. 